\title{
Left anterior hemiblock masking inferior myocardial infarction
}

\author{
Natalio Cristal, Winston Ho, and Mosche Gueron \\ From the Coronary Care Unit, Soroka Medical Center, Beersheva, Israel
}

A case is presented in which transient left anterior hemiblock masked the electrocardiographic signs of old inferior wall infarction. The electrocardiographic features are correlated with the anatomopathological findings. This case re-emphasizes the need for careful evaluation of patients with chest pain and left anterior hemiblock.

The well-documented observation that left anterior hemiblock is capable of mimicking, augmenting, or concealing the electrocardiographic signs of coexisting inferior wall infarction (Rosenbaum et al., 1972) acquires extreme significance when it is considered that this hemiblock is one of the most common intraventricular conduction abnormalities to be found in an out-patient population (Rosenbaum, Elizari, and Lazzari, I968), or to develop in patients with acute myocardial infarction (Marriott and Hogan, 1970).

It has been postulated that the anatomical relation between the necrotic area and the ventricular site first depolarized during left anterior hemiblock will ultimately determine the electrocardiographic pattern resulting from the combination of the hemiblock and the inferior myocardial infarct. (Rosenbaum et al., I968.) So far, however, this hypothesis has not been confirmed by anatomical observations.

The purpose of this communication is to correlate the electrocardiographic and anatomical findings in a patient with a recent septal infarct in whom a transient left anterior hemiblock masked the pattern of previous inferior wall infarction.

\section{Case report}

A 6I-year-old man was admitted to hospital for severe retrosternal pain radiating to the left shoulder and arm. He had sustained a myocardial infarct 2 years previously. The admission electrocardiogram showed normal PR and QRS intervals. The mean QRS axis in the frontal plane was $+20^{\circ}$ and there were large $Q$ waves in leads II, III, and aVF, with deep $T$ waves, findings compatible with an old myocardial infarct. The praecordial leads showed disphasic $T$ waves in $V_{I}$ to $V_{4}$ with prominent $U$ waves, as well as a $Q$ wave and flat $T$ wave in $V_{4}$ and V6 (Fig. $I$ ). A second recording (not shown) taken a few hours later showed changes consistent with an acute anteroseptal myocardial infarct. The serum enzymes confirmed the diagnosis of acute necrosis.

The patient subsequently sustained several short bouts of ventricular tachycardia abolished with lignocaine. A few hours after admission a rate-dependent right bundle-branch block developed and persisted for 24 hours. A demand pacemaker was installed. On the third hospital day the electrocardiogram showed distinct changes - the mean QRS axis in the frontal plane was directed superiorly and to the left $\left(-75^{\circ}\right)$, the QRS duration was $0.12 \mathrm{~s}$, and the PR interval $0.20 \mathrm{~s}$. Small r waves and prominent $S$ waves were present in leads II, III, and aVF. These changes were interpreted as consistent with the development of left anterior hemiblock. At this time, the changes indicating old inferior myocardial infarction were no longer present (Fig. 2). The hemiblock was transient and disappeared on the seventh hospital day, with the reappearance of the electrocardiographic signs of the old inferior infarct (Fig. 3).

Three weeks after admission, ventricular fibrillation occurred during an episode of chest pain. Resuscitation was unsuccessful. At necropsy a recent myocardial infarct was found, involving the interventricular septum and extending to the apex and lateral wall of the left ventricle. An aneurysm was present at the apex with a large thrombus adherent to the septal portion of the myocardial infarct. In addition, there was extensive fibrosis involving the posterior diaphragmatic portion of the left ventricle, consistent with old myocardial infarction. This fibrosis did not involve the posterior papillary muscle and the entire adjacent area, including 
NI
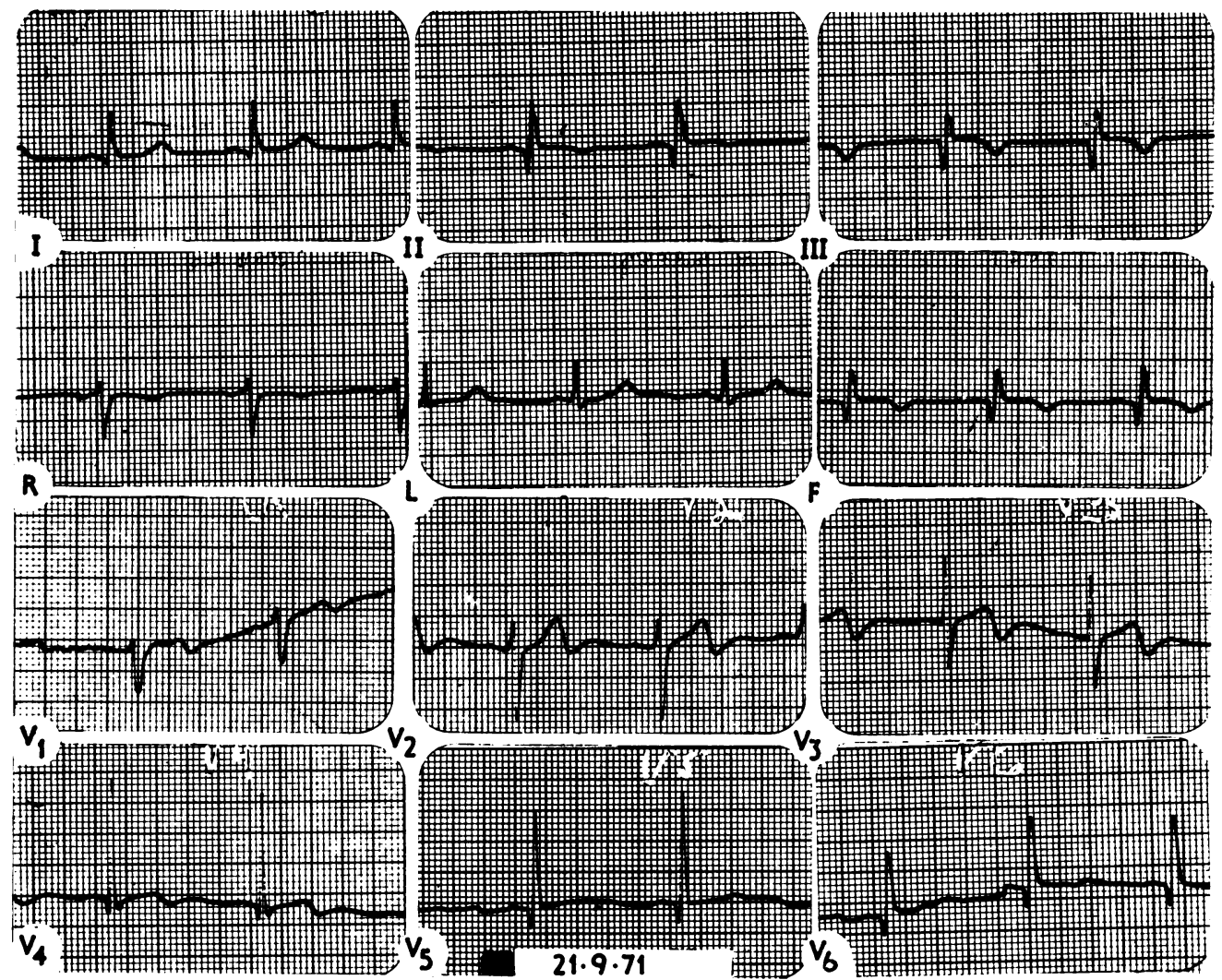

FIG. I Recording obtained on admission. The changes in the praecordial leads suggest acute anteroseptal myocardial infarction. The mean $Q R S$ axis is normal; the electrocardiogram is also diagnostic of an old inferior infarct.

the posterior basal portion of the interventricular septum (Fig. 4).

\section{Discussion}

Myers (1956), in reporting the anatomopathological findings in patients with 'horizontal hearts with small $r$ waves in AVF', was surprised at the discovery of necrotic areas in the inferior wall in 22 out of 35 cases. Later, Rosenbaum et al. (1968, 1972) focused attention on the fact that left anterior hemiblock may obscure the signs of inferior myocardial infarction by changing the characteristic $Q$ waves in leads II, III, and aVF to small $r$ waves, similar to the way in which the left bundle-branch block masks septal infarction by changing the polarity of the septal vector. They postulated that the topographical relations between the necrotic area and the zone where the impulse emerges at the end fibres of the posterior hemibundle could be decisive in concealing or augmenting the $\mathrm{Q}$ waves of inferior wall necrosis: if the necrotic area was small or located away from the base of the posterior papillary muscle, the impulse emerging from the posterior hemibundle would find enough junctional muscle present to activate, and a positive deflection would replace the $Q$ wave diagnostic of inferior necrosis. The counterpart of this situation is to be found in the study of Castellanos et al. (197I) in which the QS pattern in lead II of the scalar electrocardiogram and the absence of the first vector pointing inferiorly in the frontal plane in the vectorcardiogram were interpreted as a loss of the ventricular muscle adjacent to the end fibres of the left posterior hemibundle. As far as we know, pathological documentation has not yet been reported confirming either of these situations.

In the present case, the transient nature of the left anterior hemiblock clearly demonstrated the 

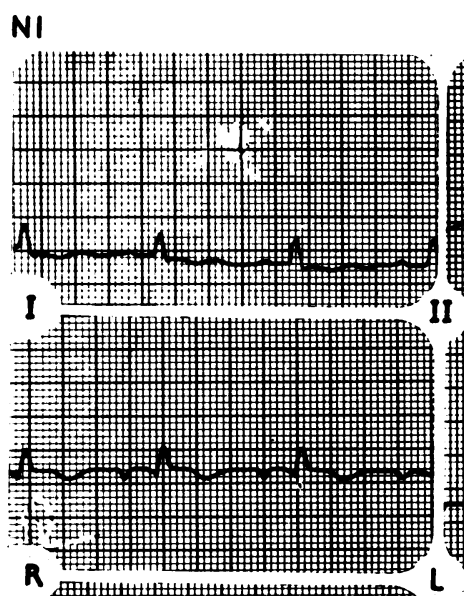
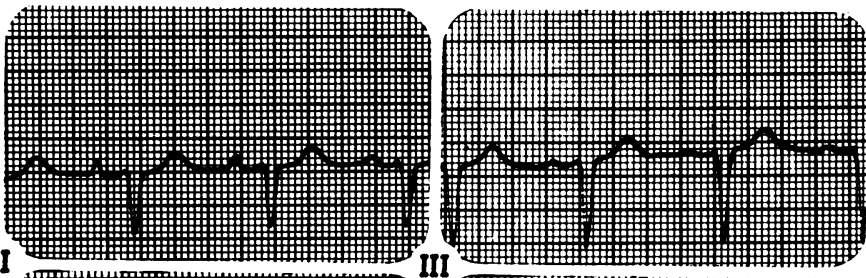

III

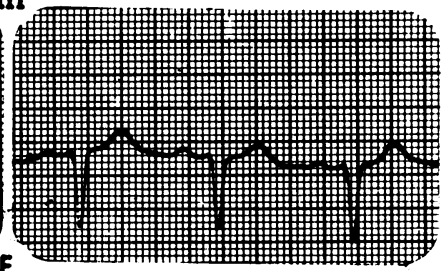

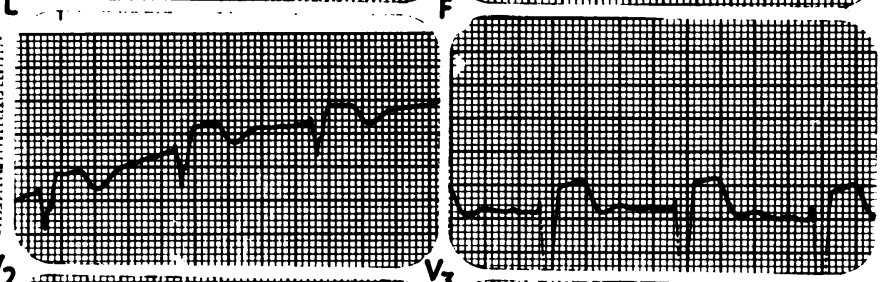

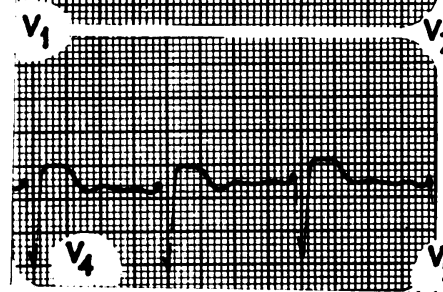

23.9.71
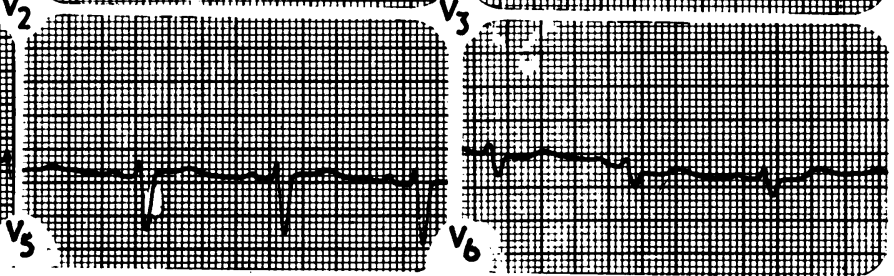

FIG. 2 Electrocardiogram on the third hospital day. The mean $Q R S$ axis is directed superiorly and to the left, changes consistent with left anterior hemiblock. A small $r$ wave is present in leads II, III, and aVF; the T waves in these leads are now positive. 


\section{NI}
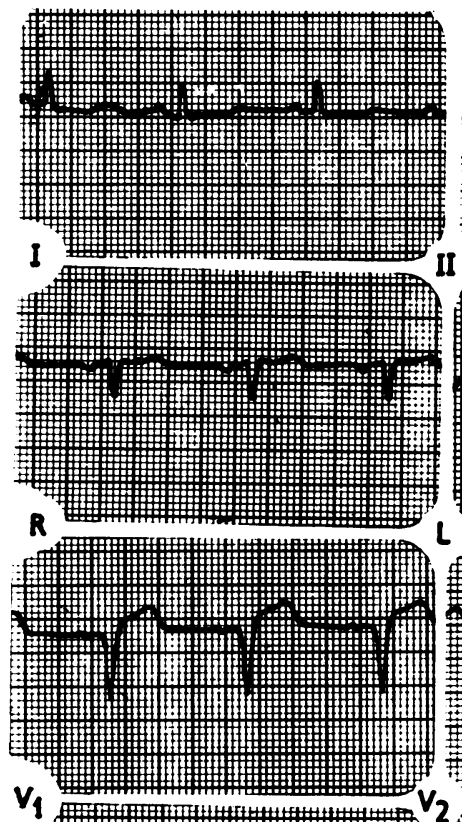

(1)

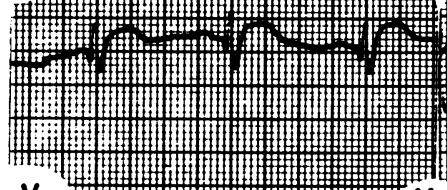

$V_{4}$

27.9 .71
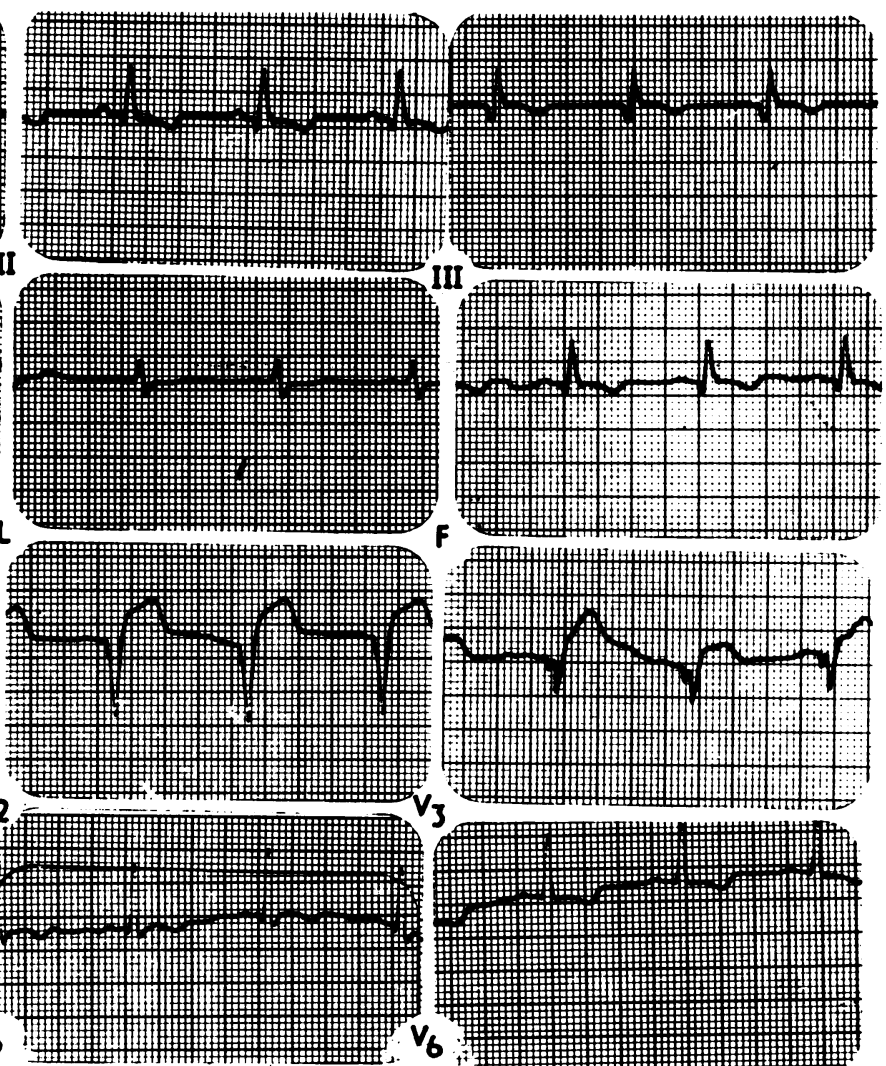

FIG. 3 Tracing on the seventh day. The left anterior hemiblock is no longer present, the evidence of the old inferior infarct is obvious. 


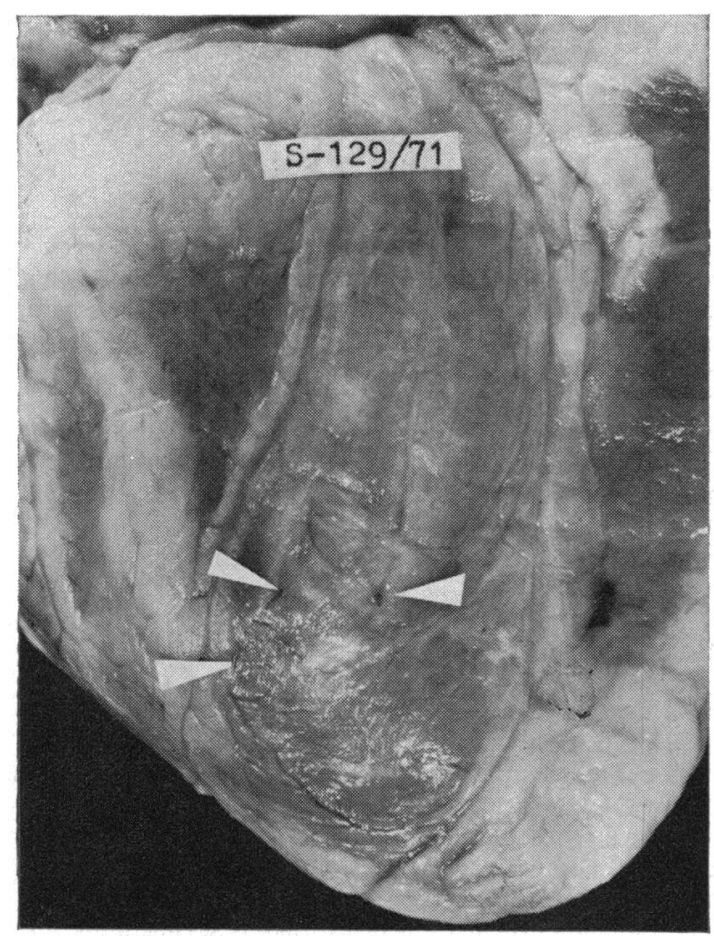

FIG. 4 Posterior inferior aspect of the left ventricle. The arrows delineate the external projection of the base of the posterior papillary muscle. A fibrotic scar is found above the arrows with healthy myocardium present in the area adjacent to the posterior papillary muscle.

changes that can be produced by the conduction defect in the electrocardiographic pattern of inferior wall infarction. The characteristic $Q$ waves were replaced by small $r$ waves, deep $S$ waves developed in the same leads, and the repolarization changes were no longer present. Altieri and Schaal (1973) reported a similar case where transient left anterior hemiblock masked the electrocardiographic evidence of inferior and anteroseptal myocardial infarction.

The anatomical findings in our case confirmed the hypothesis put forward by Rosenbaum et al. (1968): the left posterior papillary muscle and adjacent area were found to be healthy and able to undergo depolarization when the impulse reached them through the unblocked posterior division of the left bundle.

It is evident that when left anterior hemiblock is transient or intermittent, the diagnosis of a coexisting inferior infarct will soon be possible. An obvious problem exists in cases of permanent left anterior hemiblock (which is a relatively frequent electrocardiographic finding in subjects over the age of 50) in which the conduction defect may permanently obscure a necrosis in the inferior wall.

Vectorcardiography has proved to be more helpful in evaluating patients with pronounced left axis deviation, and characteristic vectorcardiographic patterns have been described for left anterior hemiblock, inferior wall infarction, and coexisting double pathologies (Castellanos, 197I; Benchimol and Desser, 1972; Benchimol, Desser, and Schumacher, 1972).

In our opinion, the case presented re-emphasizes the need for a most careful evaluation of patients presenting with suggestive chest pain together with left anterior hemiblock in the electrocardiogram.

\section{References}

Altieri, P., and Schaal, S. F. (1973). Inferior and anteroseptal myocardial infarction concealed by transient left anterior hemiblock. Fournal of Electrocardiology, 6, 257.

Benchimol, A., and Desser, K. B. (1972). Co-existing left anterior hemiblock and inferior wall myocardial infarction. American fournal of Cardiology, 29, 7.

Benchimol, A., Desser, K. B., and Schumacher, J. (1972). Value of the vectorcardiogram for distinguishing left anterior hemiblock from inferior infarction with left axis deviation. Chest, 6r, 74.

Castellanos, A. (I97I). Diagnosis of left anterior hemiblock and left posterior hemiblock in the presence of inferior wall myocardial infarction. Bulletin of New York Academy of Medicine, 47, 923.

Castellanos, A., Chahine, R. A., Chapunoff, E., Gomez, J., and Portillo, B. (1971). Diagnosis of left anterior hemiblock in the presence of inferior wall myocardial infarction. Chest, 60, 543.

Marriott, H. J. L., and Hogan, P. (1970). Hemiblock in acute myocardial infarction. Chest, 58, 342 .

Myers, G. B. (1956). The Interpretation of the Unipolar Electrocardiogram. C. V. Mosby, St. Louis, Mo.

Rosenbaum, M. B., Elizari, M. V., and Lazzari, J. O. (1968). Los Hemibloqueos. Ed. Paidos, Buenos Aires.

Rosenbaum, M. B., Elizari, M. V., Lazzari, J. O., Nav, G. S., Halpern, M. S., and Levi, R. J. (1972). In Advances in Electrocardiography. Ed. by R. C. Schlant and J. W. Hurst. Grune and Stratton, New York.

Requests for reprints to Dr. N. Cristal, Coronary Care Unit, Soroka Medical Center, Beersheva, Israel. 